\title{
Cross-normalization of MALDI mass spectrometry imaging data improves site-to-site reproducibility
} Supplemental Information

\author{
Tobias Boskamp ${ }^{1,2^{*}}$, Rita Casadonte ${ }^{3}$, Lena Hauberg-Lotte ${ }^{2}$, Sören Deininger ${ }^{1}$, \\ Jörg Kriegsmann ${ }^{3,4}$, and Peter Maass ${ }^{2}$ \\ ${ }^{1}$ Bruker Daltonics GmbH \& Co. KG, Bremen, Germany \\ ${ }^{2}$ Center for Industrial Mathematics, University of Bremen, Germany \\ ${ }^{3}$ Proteopath, Trier, Germany \\ ${ }^{4}$ Center for Histology, Cytology and Molecular Diagnostic, Trier, Germany \\ *Corresponding author, tobias.boskamp@bruker.com
}

2021-06-14

\begin{abstract}
This is an appendix to the manuscript "Cross-normalization of MALDI mass spectrometry imaging data improves site-to-site reproducibility" presenting a cross-normalization method that is specifically designed to operate on MALDI mass spectrometry imaging data.

The proposed method includes analyzing the statistical intensity distribution of spectral signals as a function of the corresponding masses. By comparing the intensity distributions of multiple spectra, intensity transformations are derived that normalize the spectra to a common reference.

This appendix includes a formal presentation of this method, details on the tissue samples and preparation protocols used for validation, as well as additional results and figures.
\end{abstract}




\section{Appendix}

\section{A.1 Sample preparation and data acquisition}

Preparation of the tissue samples for MALDI MSI data acquisition was performed according to optimized standard protocols as described earlier [1,4]. Serial sections $(5 \mu \mathrm{m})$ were mounted on indium tin oxide (ITO) slides (Bruker Daltonics, Bremen, Germany) and deparaffinized and rehydrated in a graded ethanol series. Antigen retrieval was performed $\left(20 \mathrm{~min}, 95^{\circ} \mathrm{C}, 10 \mathrm{mmol}\right.$ Tris buffer, $\mathrm{pH}$ 9.0) in a decloaking chamber (Biocare Medical, Pacheco, CA, USA).

We used three different protocols for on-tissue digestion, matrix deposition, and MALDI MSI data acquisition, mainly differing in the sprayer and MALDI MSI instruments being used. Details for Protocol 1 ("ImagePrep and autoflex") and Protocol 2 ("TM Sprayer and rapiflex") are given in Table S-1. The third protocol, Protocol 1a, is a combination of Protocol 2 for the sample preparation and Protocol 1 for the MALDI MSI acquisition steps ("TM Sprayer and autoflex").

Table S-1: Summary of sample preparation and MALDI MSI acquisition parameters for Protocols 1 and 2 (ABC: ammonium bicarbonate, ACN: acetonitrile, CHCA: alpha-cyano-4-hydroxycinnamic acid, TFA: trifluoroacetic acid)

\begin{tabular}{|c|c|c|}
\hline Parameter & Protocol 1 & Protocol 2 \\
\hline \multicolumn{3}{|c|}{ Sample preparation } \\
\hline Sprayer & $\begin{array}{l}\text { ImagePrep (Bruker Daltonics, Bre- } \\
\text { men, Germany) }\end{array}$ & $\begin{array}{l}\text { TM Sprayer (HTX Technologies, } \\
\text { Chapel Hill, USA) }\end{array}$ \\
\hline On-tissue digestion & $\begin{array}{l}\text { deposition of } 0.1 \mu \mathrm{g} / \mathrm{\mu l} \text { trypsin solu- } \\
\text { tion in } 40 \mathrm{mmol} \mathrm{ABC} \text { and } 10 \% \mathrm{v} / \mathrm{v} \\
\mathrm{ACN} \text {, incubation for } 2 \mathrm{~h} \text { at } 37^{\circ} \mathrm{C}\end{array}$ & $\begin{array}{l}\text { deposition of } 0.025 \mathrm{\mu g} / \mu \mathrm{l} \text { trypsin solu- } \\
\text { tion in } 20 \mathrm{mmol} \mathrm{ABC} \text {, incubation for } \\
2 \mathrm{~h} \text { at } 37^{\circ} \mathrm{C}\end{array}$ \\
\hline Matrix deposition & $\begin{array}{l}7 \mathrm{mg} / \mathrm{ml} \mathrm{CHCA} \mathrm{in} 50 \% \mathrm{v} / \mathrm{v} \mathrm{ACN} \\
\text { and } 0.5 \% \mathrm{v} / \mathrm{v} \text { TFA }\end{array}$ & $\begin{array}{l}10 \mathrm{mg} / \mathrm{ml} \mathrm{CHCA} \text { in } 70: 30 \mathrm{ACN} / \mathrm{H}_{2} \mathrm{O} \\
\text { and } 1.0 \% \mathrm{v} / \mathrm{v} \text { TFA }\end{array}$ \\
\hline \multicolumn{3}{|c|}{ MALDI MSI acquisition } \\
\hline MSI instrument & autoflex speed (Bruker) & rapiflex TissueTyper (Bruker) \\
\hline Spot spacing & $100 \mu \mathrm{m}$ & Tera 2: $50 \mu \mathrm{m}$, MDP 2: $100 \mu \mathrm{m}$ \\
\hline Mass range & $700-4000 \mathrm{~m} / \mathrm{z}$ & $600-3200 \mathrm{~m} / \mathrm{z}$ \\
\hline Calibration & \multicolumn{2}{|c|}{ external calibration using Peptide Calibration Standard II (Bruker) } \\
\hline
\end{tabular}

\section{A.2 Description of datasets}

Details of the teratoma and tumor TMA datasets investigated in this study are given in Tables S2, S-3, and S-4 below. More information on the tumor TMA datasets is also found in Cordero et al. [1].

Fig. S-1 shows hematoxylin-eosin (HE) stained sections used for datasets Tera 1a and Tera 2, together with average spectra and some selected $m / z$-images. (See Fig. 1 in the main text for corresponding images for Tera 1.) For the TMA datasets, Fig. S-2 shows average spectra of all three TTBO datasets.

Note that in particular in the teratoma datasets, the number of spots in the different classes are not balanced, which may have an adverse effect on the performance of the linear discriminant analysis (LDA) classification models. We accommodated for this by specifying the option "Prior: uniform" in the Matlab implementation of the LDA algorithm. 
Table S-2: Characteristics of the six datasets investigated in this study. 'Num. spots' and 'Num. bins' refers to the number of spectra and $m / z$ bins, resp.

\begin{tabular}{lllrr}
\hline Dataset & Sprayer & MS instrument & Num. spots & Num. bins \\
\hline Tera 1 & ImagePrep & autoflex & 23,622 & 20,992 \\
Tera 1a & TM Sprayer & autoflex & 21,385 & 20,992 \\
Tera 2 & TM Sprayer & rapiflex & 90,933 & 53,300 \\
\hline TTBO 1 HB & ImagePrep & autoflex & 42,460 & 20,883 \\
TTBO 1 TR & ImagePrep & autoflex & 43,504 & 20,883 \\
TTBO 2 & TM Sprayer & rapiflex & 48,376 & 66,726 \\
\hline
\end{tabular}

Table S-3: Teratoma datasets, numbers of spots for each tissue phenotype, dataset, and annotated cross-validation subset.

\begin{tabular}{lrrrrrrrrr}
\hline Tissue type & Tera 1 & A & B & Tera 1a & A & B & Tera 2 & A & B \\
\hline All & 23,622 & 2623 & 2564 & 21,385 & 2294 & 2309 & 90,933 & 10,664 & 10,197 \\
Bloody area & 107 & 55 & 52 & 86 & 48 & 38 & 304 & 175 & 129 \\
Cartilage & 511 & 276 & 235 & 110 & 74 & 36 & 1861 & 1038 & 823 \\
Pancreatic glands & 270 & 127 & 143 & 330 & 143 & 187 & 1373 & 587 & 786 \\
Sebaceous glands & 2839 & 1510 & 1329 & 2863 & 1450 & 1413 & 12,255 & 6299 & 5956 \\
Simple epithelium & 130 & 62 & 68 & 94 & 49 & 45 & 250 & 146 & 104 \\
Smooth muscle & 1125 & 543 & 582 & 936 & 480 & 456 & 4258 & 2233 & 2025 \\
Squamous epithelium & 205 & 50 & 155 & 184 & 50 & 134 & 560 & 186 & 374 \\
\hline
\end{tabular}

Table S-4: Tumor TMA datasets, numbers of spots for each tissue phenotype, dataset, and annotated cross-validation subset.

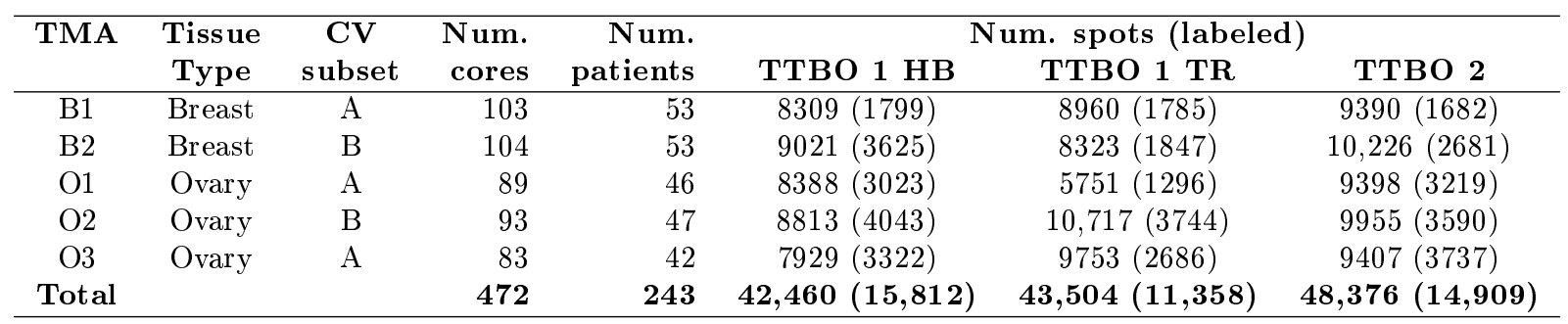



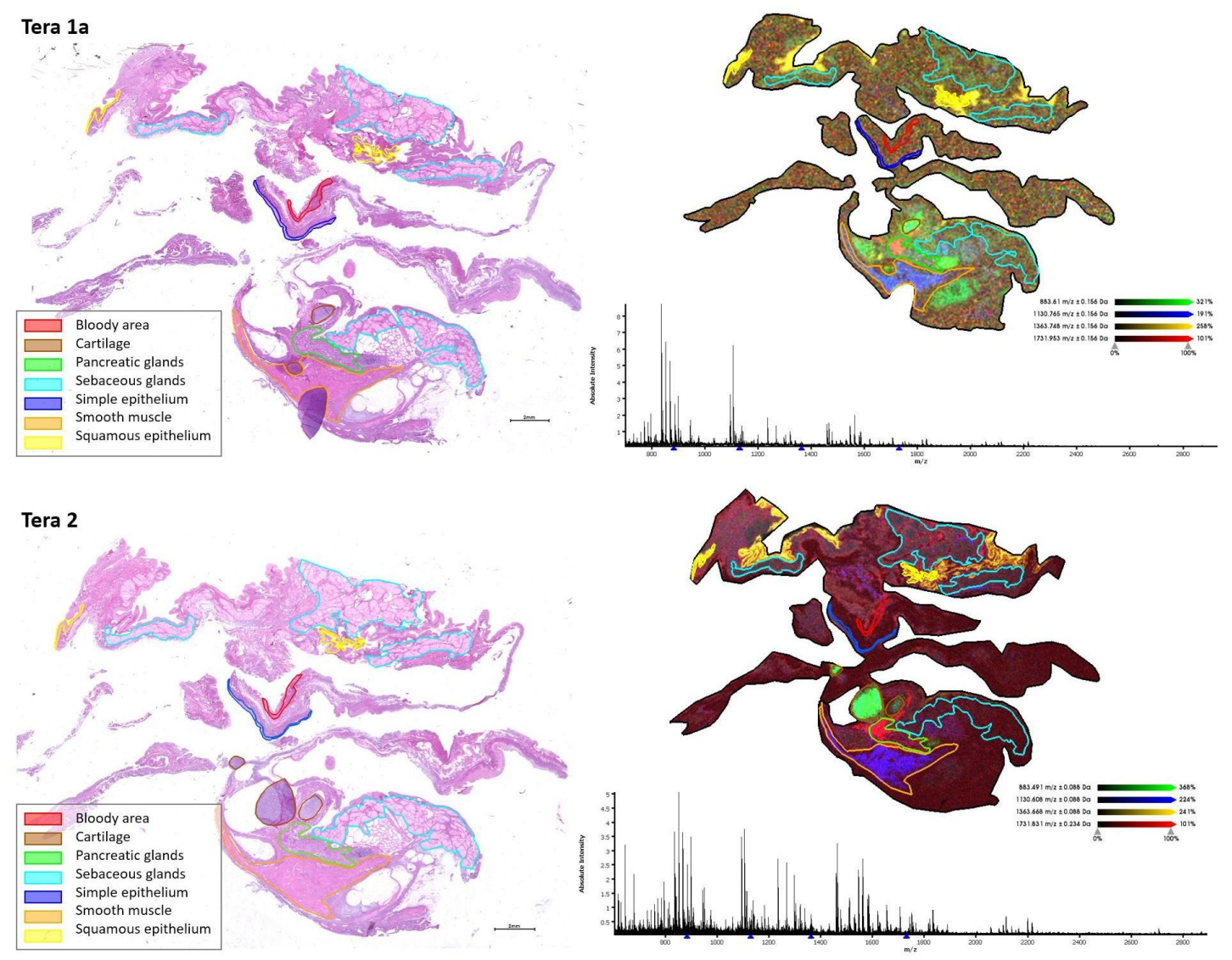

Figure S-1: For datasets Tera 1a (top) and Tera 2 (bottom), hematoxylin-eosin (HE) stained sections with histological annotations for seven different tissue phenotypes (left), as well as overall mean spectra and color representations of four $\mathrm{m} / \mathrm{z}$ images with apparent spatial correlation to different tissue phenotypes (right). 

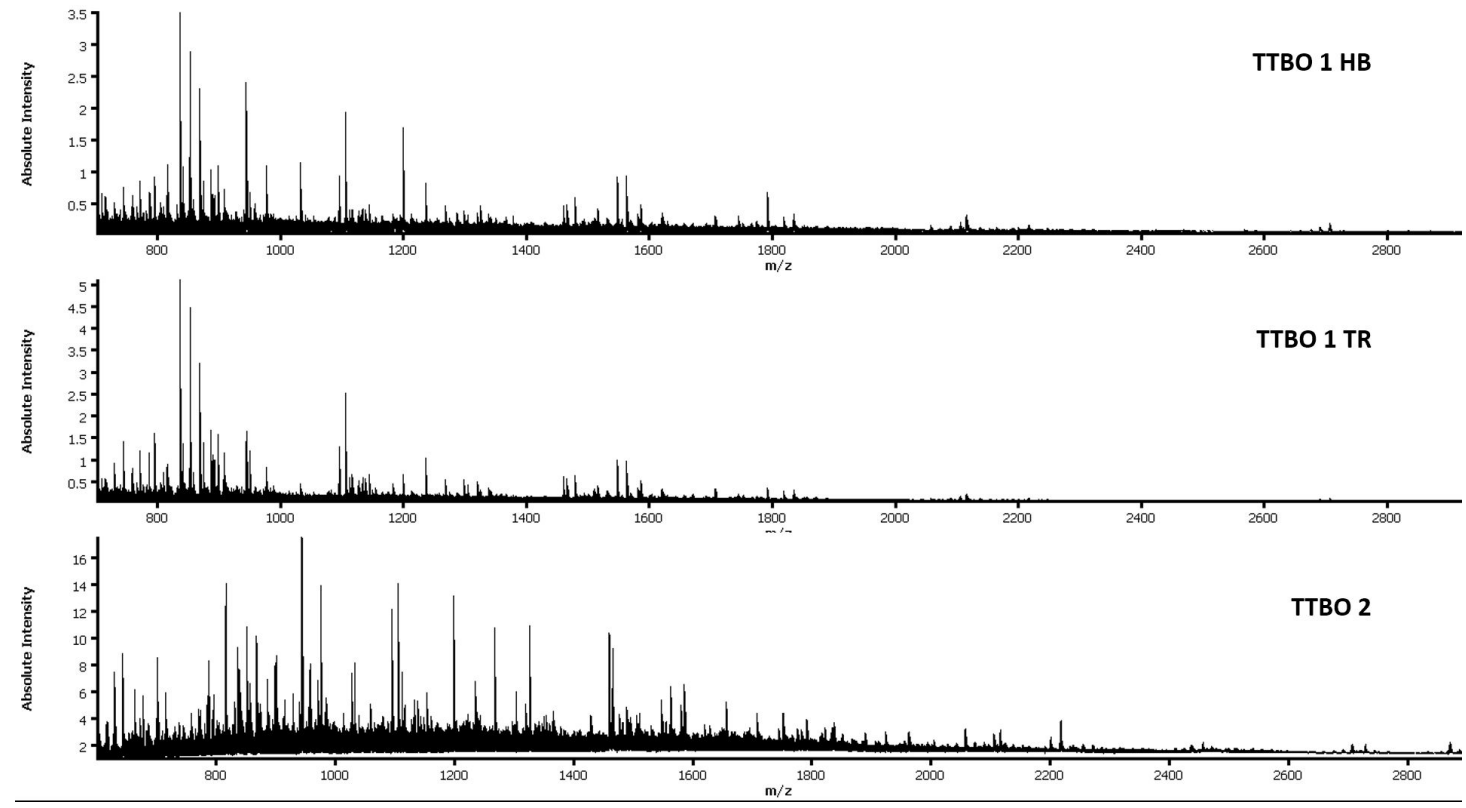

Figure S-2: Average spectra of the three TMA datasets TTBO 1 HB (top), TTBO 1 TR (middle), and TTBO 2 (bottom). 


\section{A.3 Cross-normalization based on spectral intensity profiles}

In a cross-normalization scenario, we are given a set of mass spectra that need to be transformed in such a way that their spectral intensities become comparable to each other, thus allowing a joint computational analysis of all spectra. The proposed cross-normalization method based on spectral intensity profiles consists of the following steps:

1. Compute spectral intensity profiles for each of the individual mass spectra.

2. Compute a reference profile from the individual intensity profiles.

3. Transform each spectrum, such that its intensity profile becomes equal to the reference profile.

Note that the third step is also applicable to spectra that were not involved in computing the reference profile in the previous step.

\section{A.3.1 Spectral intensity profiles}

In the first step, spectral intensity profiles are computed that describe the statistical intensity distribution of spectral signals as a function of the corresponding masses.

Let a single spectrum be given as $S=\left(s_{i}, m_{i}\right)_{i=1 \ldots N}$, consisting of the $N$ intensities $s_{1} \ldots s_{N}$ at $m / z$ locations $m_{1}<\ldots<m_{N}$. Since spectral intensities are non-negative, we have $s_{i} \geq 0$.

Furthermore, let a set of disjoint mass intervals $I_{j} \subset\left[m_{1}, m_{N}\right](j=1 \ldots M)$ be given, covering the full mass range without overlap. Typically, one would choose between two and five equally sized intervals.

We are interested in the statistical distributions of the intensity values $s_{i}$ falling into the different intervals $I_{j}$. The corresponding empirical cumulative distribution functions $F_{j}$ are given by

$$
F_{j}(x)=\frac{1}{\left|N_{j}\right|}\left|\left\{i \in N_{j}: s_{i} \leq x\right\}\right|,
$$

where $N_{j}=\left\{1 \leq i \leq N: m_{i} \in I_{j}\right\}$ denotes the set of indices $i$ for which the corresponding $m / z$ value $m_{i}$ falls into interval $I_{j}$. Thus, for any possible intensity value $x, F_{j}(x)$ yields the proportion of spectral intensities in interval $I_{j}$ not exceeding $x$.

For the purpose of comparing different intensity distributions, it is beneficial to consider the quantile functions $F_{j}^{-1}$, given by

$$
F_{j}^{-1}(p)=\inf \left\{x \in \mathbb{R}: F_{j}(x) \geq p\right\}
$$

for any probability $p \in[0,1]$. In other words, $F_{j}^{-1}(p)$ yields the spectral intensity below which the proportion $p$ of intensity values in interval $I_{j}$ is found.

Representing the intensity distributions by quantile functions has the advantage that their support always lies within $[0,1]$, regardless of the respective intensity range of $S$. This allows us to approximate the $F_{j}^{-1}$ by the quantile values assumed for some fixed, discrete values of $p$.

More specifically, let $P=\left(p_{0}, \ldots, p_{K}\right)$ be a quantile scale, i.e. a tuple of probability values $p_{k}$ with $0<p_{0}<\ldots<p_{K}<1$. We denote the corresponding quantile values for spectrum $S$ by $\varphi(S)$, where

$$
\varphi(S)=\left(\varphi_{j, k}\right)_{j, k}, \quad \text { with } \quad \varphi_{j, k}=F_{j}^{-1}\left(p_{k}\right) \quad \text { for } \quad j=1 \ldots M, k=0 \ldots K .
$$

In other words, $\varphi(S)$ represents a matrix of entries $\varphi_{j, k}$, where the $j$-th row corresponds to mass interval $I_{j}$, and the $k$-th column to the $p_{k}$-quantiles. 
In practice, mass spectra are often represented by relative intensity values, where the absolute scale of reference is not known. Thus, two spectra $S$ and $S^{\prime}$ are considered equivalent if they only differ by some constant scaling factor, i.e. $S=c S^{\prime}$ for some $c>0$.

This motivates the definition of the intensity profile $Q$ of spectrum $S$ as some form of logarithmic derivative of $\varphi(S)$. Thus, $Q$ is given by the matrix $\left(Q_{j, k}\right)$,

$$
Q_{j, k}=\log \left(\varphi_{j, k}\right)-\log \left(\varphi_{j, k-1}\right)
$$

for $j=1 \ldots M, k=1 \ldots K$. As is easily seen, $Q$ is invariant under scaling of $S$, i.e.

$$
Q(c S)=Q(S) \text { for any } c>0 .
$$

Note that the above definition requires that all $\varphi_{j, k}$ for $k=0 \ldots K$ are positive. As long as spectrum $S$ is not identical zero, this can always be achieved by an appropriate choice of the quantile scale $P$. For the purpose of cross-normalization, however, $P$ may be fixed before all spectra $S$ are known, in which case $\varphi_{j, k}(S)=0$ may occur for some $j, k$, and $S$.

Since $0 \leq \varphi_{j, k-1} \leq \varphi_{j, k}$, equation (4) is well defined if and only if $\varphi_{j, k-1}>0$. Thus, we consider those $Q_{j, k}$ with $\varphi_{j, k-1}=0$ as undefined.

\section{A.3.2 Reference profile}

For computing a reference profile, we consider a set of spectra $\left(S^{t}\right)_{t=1 \ldots T}$ for some $T>1$. (For $T=1$, the spectral intensity profile of $S^{1}$ represents the reference spectrum, and this step becomes trivial.)

Using equations (3) and (4), we compute the quantile values $\varphi\left(S^{t}\right)$ and intensity profiles $Q\left(S^{t}\right)$ for all spectra $S^{t}$. Denoting by $T_{j, k}^{*}$ the set of all indices $t$ for which $Q_{j, k}\left(S^{t}\right)$ is defined,

$$
T_{j, k}^{*}=\left\{t \in\{1 \ldots T\}: \varphi_{j, k-1}\left(S^{t}\right)>0\right\},
$$

we form the reference profile $\bar{Q}$ by taking the arithmetic mean of the individual intensity profiles,

$$
\bar{Q}_{j, k}=\frac{1}{\left|T_{j, k}^{*}\right|} \sum_{t \in T_{j, k}^{*}} Q_{j, k}\left(S^{t}\right) .
$$

Note that this definition requires that the $T_{j, k}^{*}$ are all non-empty. In fact, $T_{j, k}^{*}=\emptyset$ implies that $\varphi_{j, \nu}\left(S^{t}\right)=0$ for all $0 \leq \nu<k$ and for all spectra $S^{t}$, indicating that the choice of the quantile scale $P$ is not appropriate for the given set of spectra.

\section{A.3.3 Intensity transformation}

In the last step, given the reference profile $\bar{Q}$ and a spectrum $S=\left(s_{i}, m_{i}\right)$, we define an intensity transformation $g$ mapping $S$ to the transformed spectrum

$$
\tilde{S}=\left(g\left(s_{i}, m_{i}\right), m_{i}\right),
$$

such that the spectral intensity profile of the transformed spectrum equals the reference profile,

$$
Q(\tilde{S})=\bar{Q} .
$$

This is done by reverting equation (4) to obtain reference quantile values $\bar{\varphi}_{j, k}$,

$$
\begin{aligned}
& \bar{\varphi}_{j, k}=\exp \left(\sum_{\nu=1}^{k} \bar{Q}_{j, \nu}\right) \text { for } k \geq 1, \\
& \bar{\varphi}_{j, 0}=1 .
\end{aligned}
$$


Next, for each mass interval $I_{j}$ we define an interpolating function $g_{j}: \mathbb{R}_{\geq 0} \rightarrow \mathbb{R}_{\geq 0}$ mapping the spectrum's quantile values $\varphi_{j, k}(S)$ to the reference values $\bar{\varphi}_{j, k}$, extrapolating outside $\left(\varphi_{j, 0}, \varphi_{j, K}\right)$ by constant continuation,

$$
\begin{aligned}
g_{j}\left(\varphi_{j, k}\right)=\bar{\varphi}_{j, k} & \text { for } k=0 \ldots K, \\
g_{j}(x)=\bar{\varphi}_{j, 0} & \text { for } x<\varphi_{j, 0}, \text { and } \\
g_{j}(x)=\bar{\varphi}_{j, K} & \text { for } x>\varphi_{j, K} .
\end{aligned}
$$

We use spline interpolation to define $g_{j}(x)$ for $\varphi_{j, k-1}<x<\varphi_{j, k}$, but obtained very similar results using linear interpolation.

Finally, the above functions $g_{j}$ are combined into an overall intensity transformation $g$ by a further interpolation step. Denoting by $\bar{m}_{j}$ the center of the mass interval $I_{j}$, we define the transformation $g$,

$$
g: \mathbb{R}_{\geq 0} \times \mathbb{R}_{>0} \rightarrow \mathbb{R}_{\geq 0}
$$

by linear interpolation between the $g_{j}$,

$$
\begin{aligned}
g(x, m) & =(1-\lambda) g_{j-1}(x)+\lambda g_{j}(x), \text { with } \\
\lambda & =\frac{m-\bar{m}_{j-1}}{\bar{m}_{j}-\bar{m}_{j-1}}, \quad \bar{m}_{j-1} \leq m \leq \bar{m}_{j} .
\end{aligned}
$$

Again, we use constant continuation outside the range $m \in\left(\bar{m}_{1}, \bar{m}_{M}\right)$,

$$
\begin{aligned}
& g(x, m)=g_{1}(x), \quad \text { for } m<\bar{m}_{1} \\
& g(x, m)=g_{M}(x), \quad \text { for } m>\bar{m}_{M} .
\end{aligned}
$$

To summarize, we compute intensity profiles $Q\left(S^{t}\right)$ for a set of spectra $\left(S^{t}\right)_{t}$, obtain a reference profile $\bar{Q}$ by taking the arithmetic mean of the $Q\left(S^{t}\right)$, and use this reference profile to normalize the spectra $\left(S^{t}\right)$ (and any further spectrum $S$ ). Note that the quantile scale $P$ and the partition of the mass range into the intervals $\left(I_{j}\right), j=1 \ldots M$, is the same throughout all these steps. As a consequence, all spectra need to be given over a common mass range.

\section{A.3.4 Choice of the quantile scale}

Care should be taken to make an appropriate choice of the quantile scale $P$, as it may have a strong impact on the resulting intensity transformations. From definition (4) of the intensity profile $Q$ we see that each $Q_{j, k}$ corresponds to spectral intensities in the quantile range $p_{k-1}<$ $p \leq p_{k}$. Choosing a very fine quantile scale with large $K$ may result in some of the $Q_{j, k}$ being dependent on only a few spectral intensity values, and thus strongly affected by random effects. On the other hand, we want $Q$ to approximate the statistical distribution of the spectral intensities with sufficient accuracy in particular for the higher peak intensities, which, however, represent only a small proportion of all data points.

Thus, we propose to choose the quantile scale $P$ by means of a sigmoid function, with smaller increments on the lower and - in particular - higher ends, and larger increments in between. Using the standard logistic function $\gamma(x)=\left(1+e^{-x}\right)^{-1}$ we define $P$ as

$$
p_{k}=\gamma(\alpha k+\beta) \text { for } k=0 \ldots K
$$

with suitable $\alpha, \beta$, and $K \geq 2$.

Suppose that we have fixed quantile increments $\Delta_{0}$ for the lower and $\Delta_{1}$ for the upper end, i.e.

$$
p_{0}=\Delta_{0} \quad \text { and } \quad p_{K}=1-\Delta_{1} .
$$


We require that the increments become larger towards the center of the quantile scale, and in particular

$$
p_{1} \geq 2 \Delta_{0} \quad \text { and } \quad p_{K-1} \leq 1-2 \Delta_{1},
$$

which implies an upper bound for $K$. Choosing the maximum possible $K$ leads to

$$
\begin{aligned}
K & =\left\lfloor-\frac{u_{0}+u_{1}}{\max \left(v_{0}, v_{1}\right)}\right\rfloor, \\
\alpha & =-\frac{1}{K}\left(u_{0}+u_{1}\right) \text { and } \beta=u_{0},
\end{aligned}
$$

with the $u_{0,1}$ and $v_{0,1}$ being defined through the logit function $\gamma^{-1}(p)=\log \frac{p}{1-p}$,

$$
\begin{aligned}
& u_{0,1}=\gamma^{-1}\left(\Delta_{0,1}\right), \text { and } \\
& v_{0,1}=\gamma^{-1}\left(2 \Delta_{0,1}\right)-u_{0,1} .
\end{aligned}
$$

The parameters $\Delta_{0}$ and $\Delta_{1}$, are chosen based on the spectral data under consideration. For a given set of spectra $\left(S^{t}\right)_{t=1 \ldots T}$ and a given choice of mass intervals $I_{j}$, we require that the quantile ranges $0 \leq p \leq \Delta_{0}$ and $1-\Delta_{1}<p \leq 1$ include at least five spectral data points in each interval. Moreover, $\Delta_{0}$ shall be large enough so that in all $I_{j}$ the range $0 \leq p \leq \Delta_{0}$ includes at least one non-zero intensity for at least half of the spectra.

This leads to

$$
\begin{aligned}
\Delta_{1} & =\max _{j} \frac{5}{\left|N_{j}\right|}, \text { and } \\
\Delta_{0} & =\max \left(\Delta_{1}, \Delta^{*}\right), \text { with } \\
\Delta^{*} & =\max _{j} \operatorname{median}_{t} \frac{\left|\left\{i \in N_{j}: s_{i}^{t} \leq 0\right\}\right|+1}{\left|N_{j}\right|} .
\end{aligned}
$$




\section{A.4 Detailed results}

Detailed results of all processing pipelines on all intra- and cross-protocol tasks are shown in Figures S-4 and S-5, as well as in the spreadsheet file Tables.xlsx included in the supplemental information. In addition to the "AUC hi" metric used in the main text, two additional, alternative performance metrics are included: "AUC avg" denotes the average AUC achieved using any number of features $1 \leq n \leq 50$, and "AUC lo" denotes the $25 \%$ quantile of the AUC for all $n$. A comparison between the three metrics does not reveal substantial differences that would have an impact on the conclusions drawn in the main text, suggesting that the actual choice of the performance metric is of only secondary relevance.

Note that pipelines involving the median fold change (MFC) normalization were not evaluated for cross-protocol tasks, as inter-sample MFC requires generating a reference spectrum across all datasets, which is not immediately applicable to cross-protocol scenarios.

In order to illustrate the effects of different normalization methods on the classification results, we show a comparison of the baseline method "tic-cal" and the proposed cross-normalization "cal-pmr-ipn" method on the TTBO 1 TR-HB task. For both methods, we selected the best classification model and applied it to the test data, i.e. the TTBO 1 HB dataset.

The overall accuracies on all five TMAs in the test dataset were 0.788 for the baseline method, and 0.855 for the cross-normalization method. Largest differences were seen on one of the overay tumor TMAs (P2O), where accuracies of 0.772 and 0.922 , were achieved, resp. (Fig. S-3).
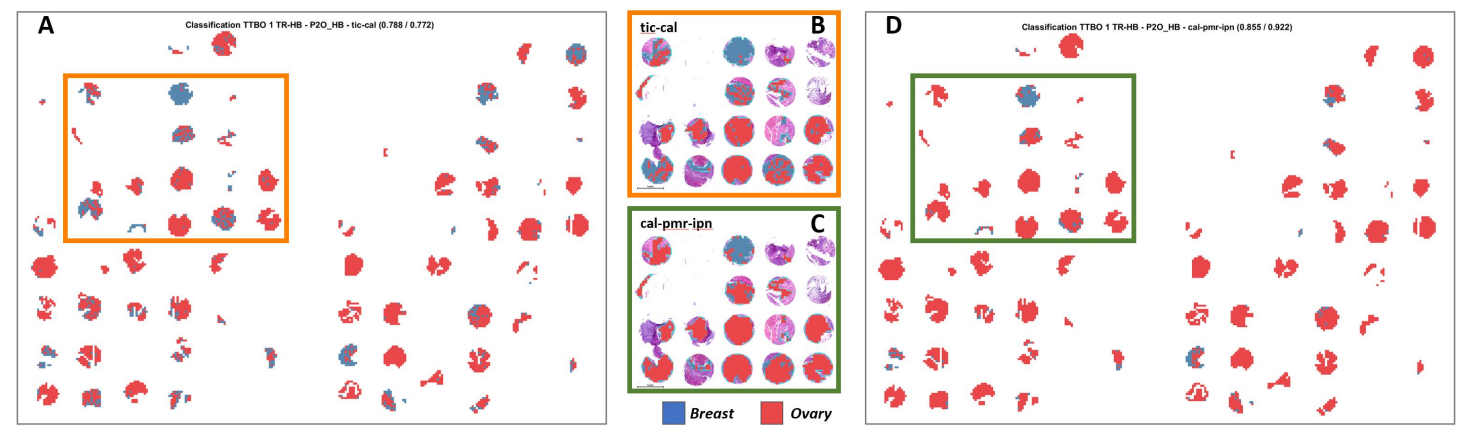

Figure S-3: Classification results on one of the ovary tumor TMAs (P2O) in the TTBO 1 TR-HB task. (A,B) Baseline method "tic-cal", (C,D) proposed cross-normalization "cal-pmr-ipn". (B,C) Closeups on a subset of cores shown as an overlay to the HE stained section. Colors indicate tissue type predictions breast (blue) and ovary (red). Since this TMA includes ovary tumor samples only, all blue spots correspond to incorrectly classified spectra. Note that due to technical limitations of the data processing pipeline involving conversion from SCiLS Lab to Matlab the TMA geometry in (A,D) slightly differs from the original. For the overlays in $(\mathrm{B}, \mathrm{C})$ this has been manually corrected. 

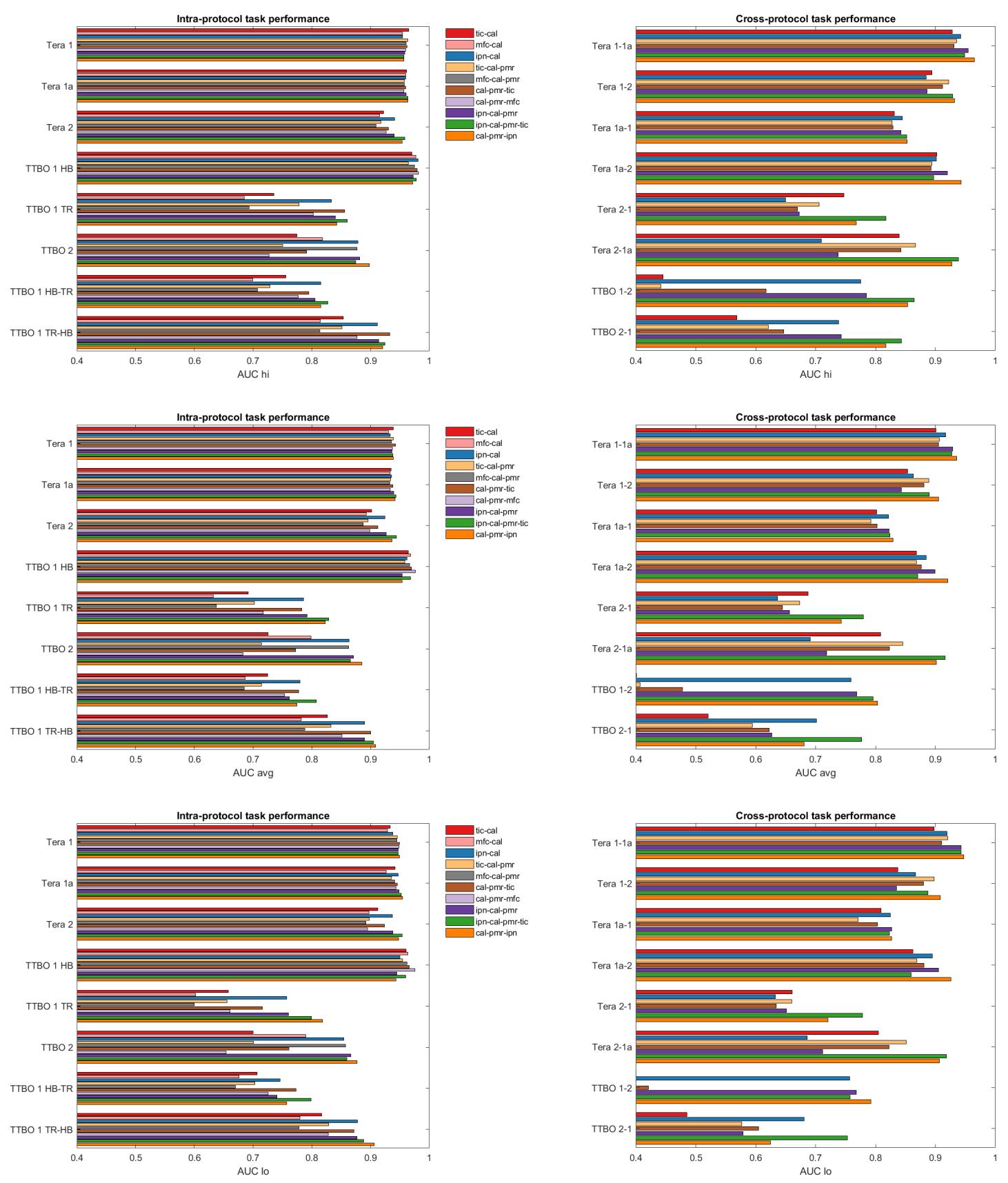

Figure S-4: Performance values obtained for all preprocessing pipelines, shown separately for the intraprotocol (left column) and cross-protocol tasks (right column). Performances are shown using the three metrics "AUC hi" (top), "AUC avg" (middle), and "AUC lo" (bottom row). 

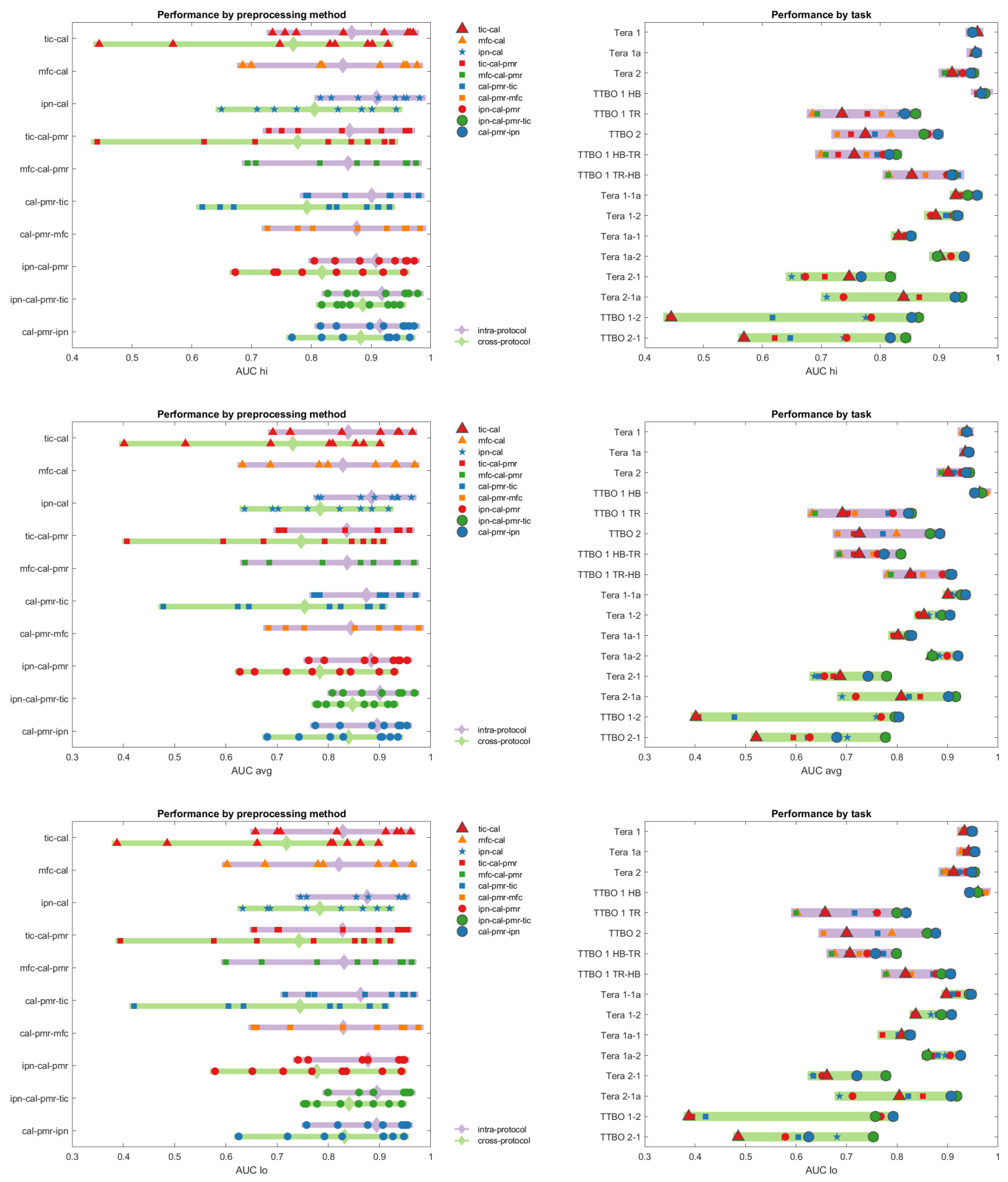

Figure S-5: Left column: Performance values obtained for all intra-protocol (blue bars) and crossprotocol (green bars) classification tasks, shown separately for each processing pipeline. Diamond markers indicate mean values. Right column: Performance values for all processing pipelines (indicated by dot color), shown separately for each task. Results of pipelines "tic-cal" (red), "ipn-cal-pmr-tic" (green), and "cal-pmr-ipn" (orange) are highlighted. Performances are shown using the the three metrics "AUC hi" (top), "AUC avg" (middle), and "AUC lo" (bottom row). Note that the top row is identical to Fig. 4 in the main text. 

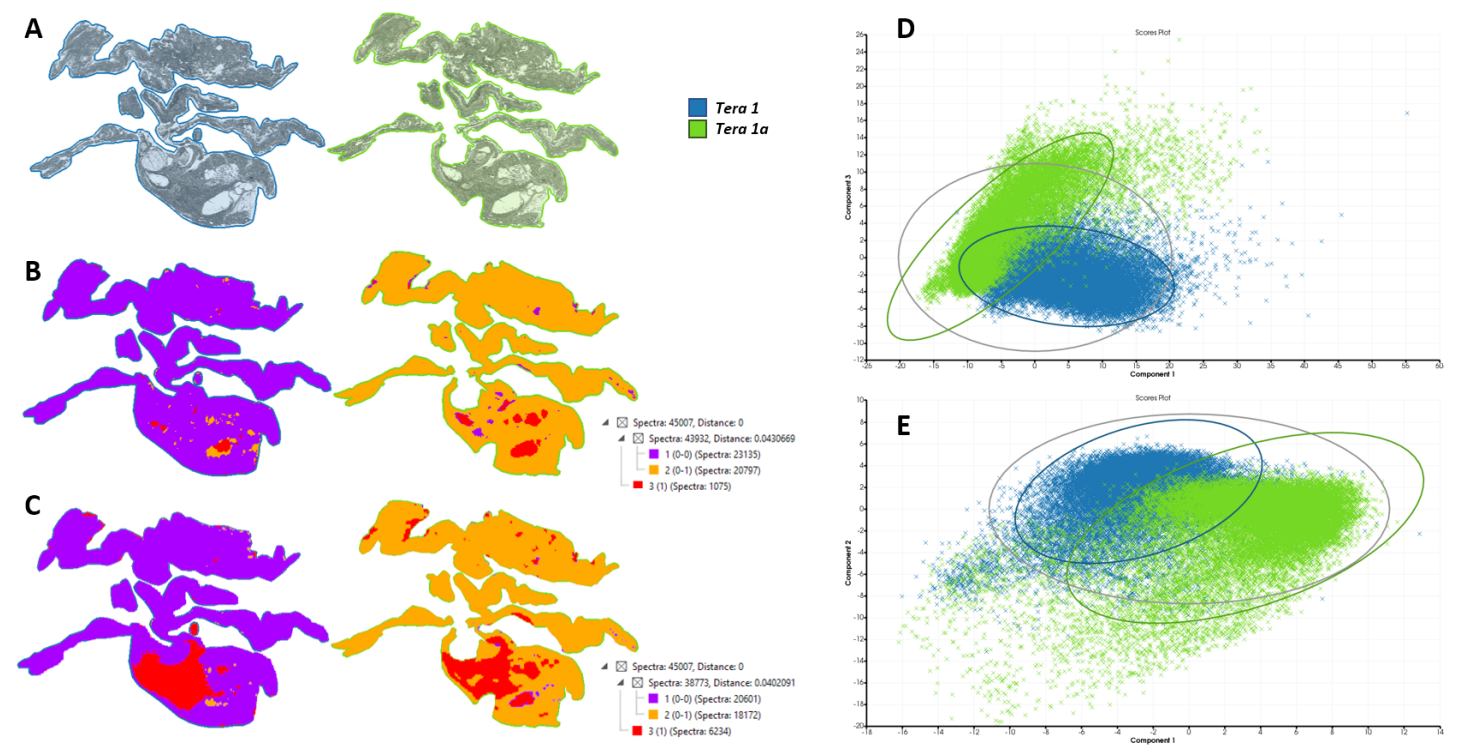

Figure S-6: Unsupervised comparison of two teratoma section datasets. (A) Optical images of sections Tera 1 (blue) and Tera 1a (green). (B) Unsupervised hierarchical clustering of both datasets after baseline processing ("tic-cal"), and (C) after cross-normalization ("cal-pmr-ipn"). (D,E) Unsupervised principal component analysis (PCA) of both datasets after baseline processing (D) and cross-normalization (E).

\section{A.5 Unsupervised analysis}

In addition to the supervised data analysis procedure described in the main text, we considered unsupervised analysis as a potentially more direct alternative to investigate the presence or absence of batch effects. In particular, hierarchical clustering and principal components analysis (PCA) are often used to assess similarities or dissimilarities of high dimensional datasets [5, 3, 2].

Our results indicate, however, that an unsupervised analysis is often too unspecific to allow a systematic investigation. By mapping the high dimensionality of the feature space to only a relatively small number of output dimensions, a large part of the information encoded in the data is unavoidably lost. Whether the remaining information accurately reflects the presence or absence of technical variation and the preservation or suppression of biological information depends on many uncontrolled factors and is hardly predictable.

To illustrate this, we present two examplary comparisons. Fig. S-6 shows the results of a comparison between datasets Tera 1 and Tera 1a (same sample, different protocols) using hierarchical clustering and PCA as implemented in the SCiLS Lab software (version 2018b, Bruker Daltonics). Clustering was performed on the baseline dataset (method "tic-cal") using the segmentation pipeline with default parameter settings, and the segmentation was broken down to three segments, demonstrating a clear separation between both datasets (Fig. S-6B). Likewise, the projection onto the first and third PCA components reveals a strong separation (Fig. S-6B), thus suggesting the presence of batch effects.

Comparing this to the results for the data obtained using cross-normalization (the "cal-pmripn" method was used), we see a very similar result from the clustering (Fig. S-6C), and a somewhat stronger overlap in the PCA (Fig. S-6E, strongest separation shown now in the first two components.) 


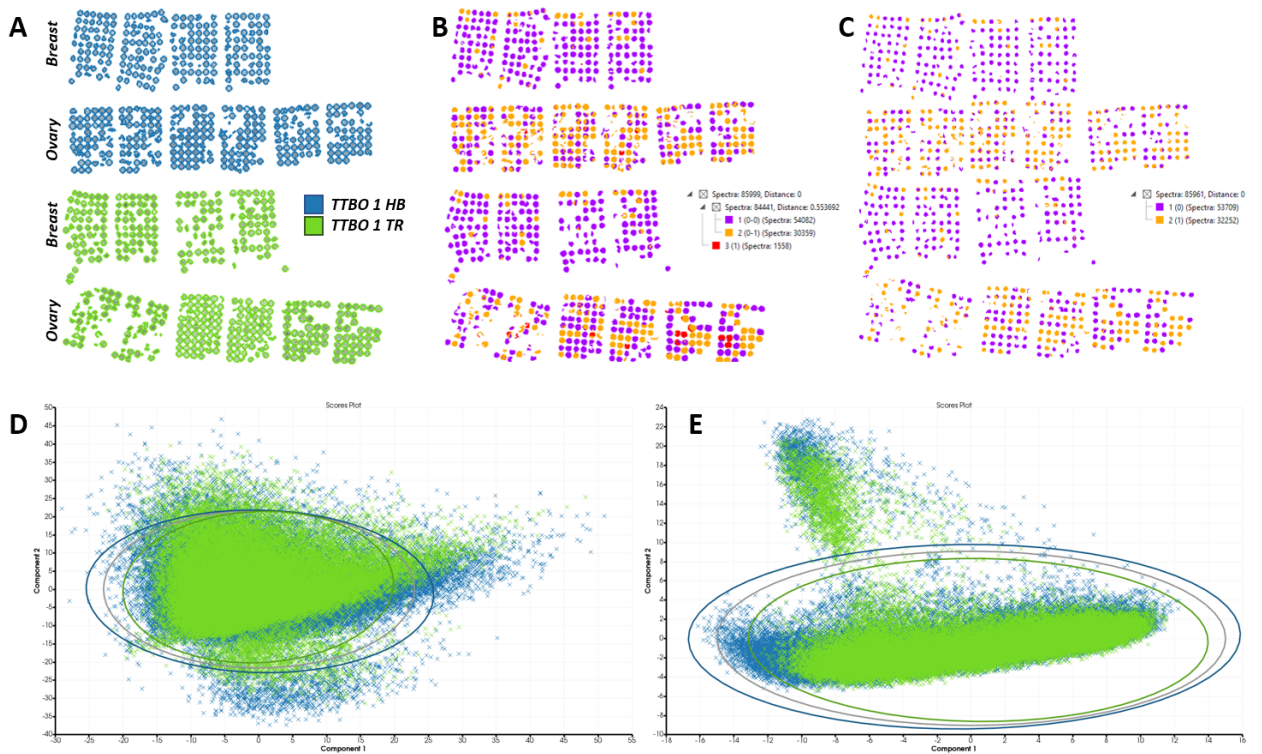

Figure S-7: Unsupervised comparison of two TMA datasets. (A) Optical images of slide sets TTBO 1 HB (blue) and TTBO 1 TR (green). (B) Unsupervised hierarchical clustering of both datasets after baseline processing ("tic-cal"), and (C) after cross-normalization ("cal-pmr-ipn"). (D,E) Unsupervised principal component analysis (PCA) of both datasets after baseline processing (D) and cross-normalization (E). Note the slightly distorted TMA geometry in (C) as compared to the original TMA layouts, which is due to technical limitations of the data processing pipeline involving conversion between SCiLS Lab and Matlab. This, however, does not have any impact on the spectral data itself.

In total, these results would suggest that a) Tera 1 and Tera 1a are heavily confounded by technical variation, and that b) the cross-normalization may have some notable effect. At least the first conclusion is in contrast to our findings using the supervised analysis (Fig. S-5), where a relatively high performance (0.93 for Tera 1-1a, 0.83 for Tera 1a-1) could already be achieved with the baseline data.

In the second example (Fig. S-7) we compare TMA datasets TTBO 1 HB and TTBO 1 TR (inter-lab scenario). The clustering seems to reveal no obvious batch effects between labs or slides, neither in the baseline data (Fig. S-7B) nor in the cross-normalized data (Fig. S-7C). The same conclusion would be drawn from the PCA results (Fig. S-7D and E). The latter additionaly reveal the presence of a subset of outlier spectra (appearing towards the bottom in graph D, towards the top in graph E), which we confirmed to be related to the muscle tissue control cores in the TMAs.

Thus, these results would suggest that a) both datasets are not confounded by technical variation, and b) cross-normalization has no positive effect. Again, this contradicts our findings from the supervised analysis, where the baseline performance is significantly lower than in the first example (TTBO 1 HB-TR: 0.76, TTBO 1 TR-HB: 0.85), and where the impact of the cross-normalization is twice as high (a gain of 0.06 , compared to 0.02 in the Tera 1/1a example above).

These results lead us to the conclusion that straight-forward unsupervised analysis tools have only limited value for investigating the presence of technical variation and for quantitatively assessing the impact of preprocessing algorithms. 


\section{References}

[1] Yovany Cordero Hernandez, Tobias Boskamp, Rita Casadonte, Lena Hauberg-Lotte, Janina Oetjen, Delf Lachmund, Annette Peter, Dennis Trede, Katharina Kriegsmann, Mark Kriegsmann, Jörg Kriegsmann, and Peter Maass. Targeted feature extraction in MALDI mass spectrometry imaging to discriminate proteomic profiles of breast and ovarian cancer. Prot Clin Appl, 13(1):1700168, 2019.

[2] Katrin Erich, Denis A. Sammour, Alexander Marx, and Carsten Hopf. Scores for standardization of on-tissue digestion of formalin-fixed paraffin-embedded tissue in MALDI-MS imaging. BBA Prot Proteom, 1865(7):907-915, 2017.

[3] Alice Ly, Rémi Longuespée, Rita Casadonte, Petra Wandernoth, Kristina Schwamborn, Christine Bollwein, Christian Marsching, Katharina Kriegsmann, Carsten Hopf, Wilko Weichert, Jörg Kriegsmann, Peter Schirmacher, Mark Kriegsmann, and Sören-Oliver Deininger. Site-to-site reproducibility and spatial resolution in MALDI-MSI of peptides from formalinfixed paraffin-embedded samples. Prot Clin Appl, 13(1):1800029, 2019.

[4] J. Oetjen, D. Lachmund, A. Palmer, T. Alexandrov, M. Becker, T. Boskamp, and P. Maass. An approach to optimize sample preparation for MALDI imaging MS of FFPE sections using fractional factorial design of experiments. Anal Bioanal Chem, 408(24):6729-40, 2016.

[5] Nico Verbeeck, Richard M. Caprioli, and Raf Van de Plas. Unsupervised machine learning for exploratory data analysis in imaging mass spectrometry. Mass Spec Rev, 39(3):245-291, 2020 . 